\title{
Clinical course of patients infected with severe acute respiratory syndrome coronavirus 2 soon after thoracoscopic lung surgery
}

Jingyu Huang, MD, PhD, ${ }^{a}$ Aifen Wang, MD, ${ }^{\mathrm{b}}$ Ganjun Kang, MD, ${ }^{\mathrm{c}}$ Dejia Li, PhD,,${ }^{\mathrm{d}, \mathrm{e}}$ and Weidong $\mathrm{Hu}, \mathrm{MD}, \mathrm{PhD}$, ${ }^{\mathrm{a}, \mathrm{f}}$ Wuhan, Hubei, and Zhangjiagang, Jiangsu, China

\footnotetext{
From the ${ }^{\mathrm{a} D e p a r t m e n t ~ o f ~ T h o r a c i c ~ S u r g e r y, ~ Z h o n g N a n ~ H o s p i t a l ~ o f ~ W u h a n ~ U n i v e r s i t y, ~ W u h a n, ~ H u b e i, ~ C h i n a ; ~}{ }^{\mathrm{b}}$ Department of Obstetrics and Gynecology, Zhangjiagang Hospital TCM Affiliated to Nanjing University of Chinese Medicine, Zhangjiagang, Jiangsu, China; ${ }^{\mathrm{c}}$ Department of Thoracic Surgery, Renmin Hospital of Wuhan University, Wuhan, Hubei, China; ${ }^{\mathrm{d}}$ Department of Preventive Health, School of Health Sciences, and ${ }^{\mathrm{e}}$ Population and Health Research Center, Wuhan University, Wuhan, Hubei, China; and ${ }^{\mathrm{f}}$ Hubei Key Laboratory of Tumor Biological Behaviors, Hubei Cancer Clinical Study Center, Wuhan, Hubei, China.

This study was funded by Zhongnan Hospital of Wuhan University Science Technology and Innovation Cultivating Fund (grant/award No. cxpy2017041) to Dr Hu.

Disclosures: The authors reported no conflicts of interest.

The Journal policy requires editors and reviewers to disclose conflicts of interest and to decline handling or reviewing manuscripts for which they may have a conflict of interest. The editors and reviewers of this article have no conflicts of interest.

Drs Huang and Wang contributed equally to this article.

Received for publication March 22, 2020; revisions received April 9, 2020; accepted for publication April 9, 2020; available ahead of print April 15, 2020

Address for reprints: Weidong Hu, MD, PhD, Department of Thoracic Surgery, ZhongNan Hospital of Wuhan University, Hubei Key Laboratory of Tumor Biological Behaviors, Hubei Cancer Clinical Study Center, 169 Donghu Rd, Wuhan, Hubei 430071, China (E-mail: huwd@whu.edu.cn).

J Thorac Cardiovasc Surg 2020;160:e91-3

$0022-5223 / \$ 36.00$

Copyright (C) 2020 by The American Association for Thoracic Surgery

https://doi.org/10.1016/j.jtcvs.2020.04.026
}

\section{CASE 1}

An 84-year-old woman with a diameter of $10 \mathrm{~mm}$ irregular subsolid nodule in the right middle lobe on computed tomography (CT) images of the chest (Figure 1, Al) was confirmed to have T1b N0 M0 lung adenocarcinoma after thoracoscopic right middle lobectomy on January 14. She had 20-year history of hypertension and diabetes and her pulmonary function testing was normal before surgery. This patient complained of cough, expectoration, and dyspnea the next day but no any signs of pneumonia displayed on her chest CT images (Figure 1, A2). On the third day after surgery, her lymphocyte count decreased from $1.93 \times 10^{9} / \mathrm{L}$ to $0.44 \times 10^{9} / \mathrm{L}$. Six days after operation, the patient complained of aggravated dyspnea, fatigue, and fever with a temperature of $37.6^{\circ} \mathrm{C}$ and $\mathrm{CT}$ displaying multiple bilateral ground-glass opacities (GGOs) (Figure 1, A3). On January 23, Reverse transcription polymerase chain reaction (RT-PCR) test for severe acute respiratory syndrome coronavirus 2 (SARS-CoV-2) was positive. On January 25, the patient developed respiratory failure, with low oxygen saturation under high flow oxygen therapy. She was administered biapenem, linezolid, and oseltamivir, and oxygen therapy. The next day, her oxygen saturation suddenly dropped to $60 \%$, and she was urgently intubated and supported by

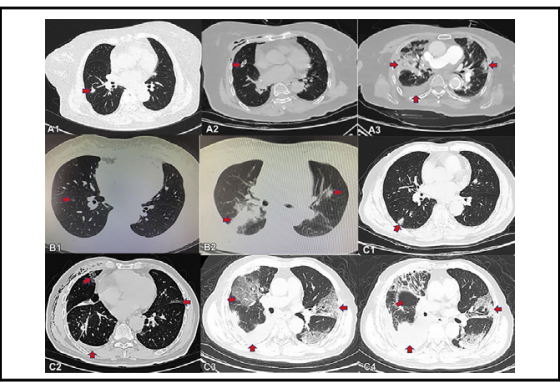

Perioperative chest computed tomography manifestation of 3 patients.

CENTRAL MESSAGE

Mortality may be very high in patients who contract SARSCoV-2 pneumonia after lung lobectomy. Lung surgery should be performed with extreme caution in SARS-COV-2 epidemic

outbreak areas.

See Commentaries on pages e95 and e97.

ventilator. Her condition deteriorated quickly and she died on January 31.

\section{CASE 2}

A 55-year-old woman with GGOs in right lower lobe (Figure 1, Bl) was suspected of early lung cancer and underwent right lower lobectomy on January 17 . She had no any morbidity and her pulmonary function testing was normal before surgery. Pathologic diagnosis was a pulmonary meningothelial-like nodule and atypical adenomatoid hyperplasia. Her lymphocyte count $\left(0.82 \times 10^{9} / \mathrm{L}\right)$ decreased on the same day. On January 20, she complained of serious cough and fever with temperature of $38.9^{\circ} \mathrm{C}$, and her CT showed bilateral pneumonia (Figure 1, B2). On January 22, RT-PCR test for SARS-CoV-2 was positive. She was transferred to an isolated ward and given ganciclovir and oseltamivir. On January 25, she suddenly presented severe dyspnea and died before intubation. 


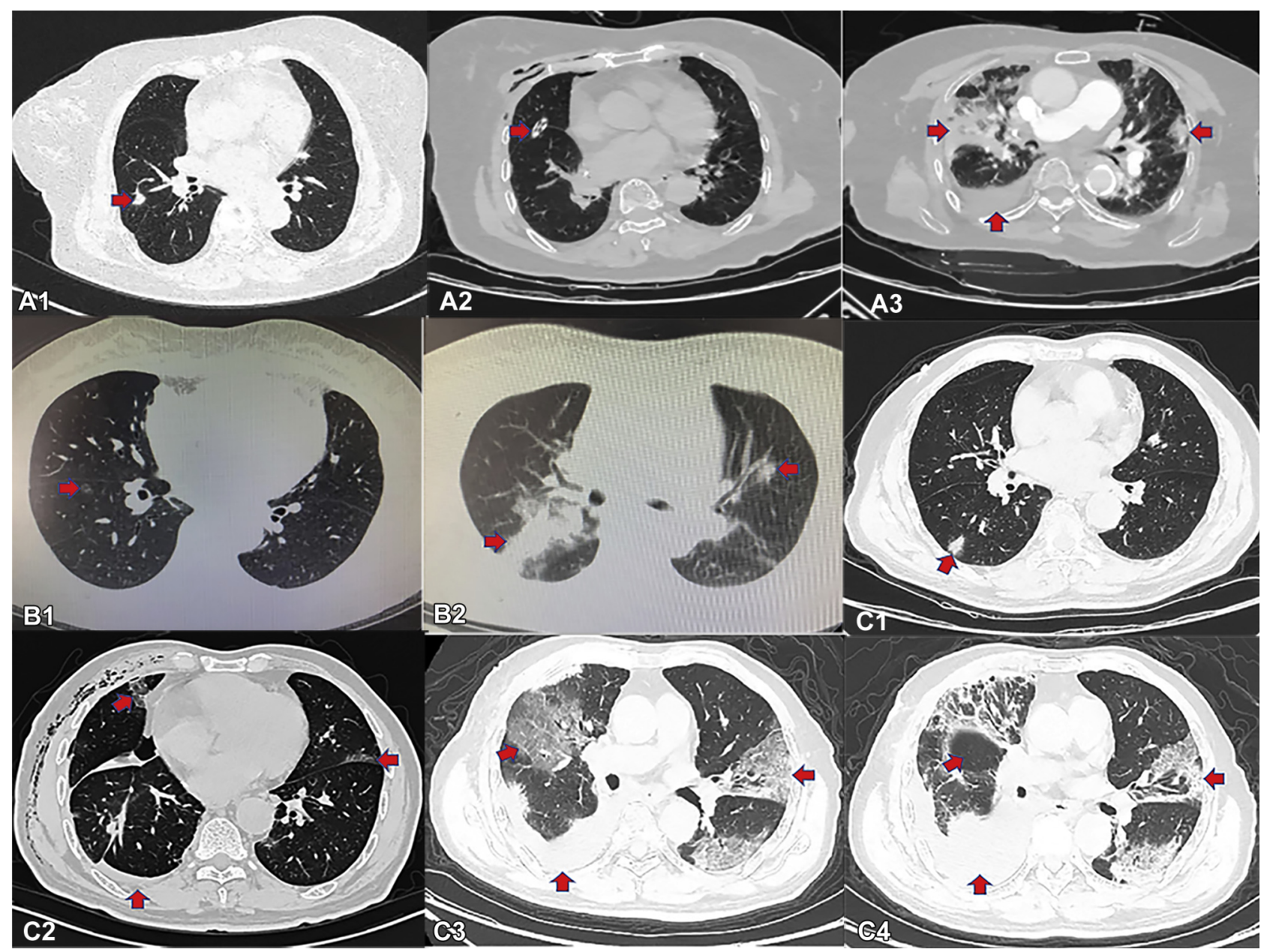

FIGURE 1. Perioperative chest computed tomography manifestation of 3 patients with lung tumor underwent thoracoscopic lobectomy. A1, Mixed ground glass opacities (GGOs) located in the right middle lobe (7 days before surgery) in Case 1. A2, No inflammation signs were found in bilateral lung, and a drainage tube was marked by a red arrow (1 day after surgery) in Case 1. A3, Multiple bilateral GGOs, especially subpleural, prominent in the right side were seen. Some fluid was found in the right thoracic cavity (6 days after surgery) in Case 1. B1, A small GGO located in the right lower lobe and suspected as malignancy (before surgery) in Case 2. B2, Large patches of high-density shadow with GGO around in the right lung. Other small GGOs were found in the left lung, some in the subpleural site (4 days after surgery) in Case 2. C1, A solid nodule located in the right lower lobe (before surgery) in Case 3. C2, Multiple bilateral subpleural GGOs were observed. Some fluid was found in the right thoracic cavity (5 days after surgery) in Case 3. C3, Multiple bilateral subpleural GGOs were getting wider and extended to bilateral hilar. The fluid in the right thoracic cavity was increasing (10 days after surgery) in Case 3 . C4, The multiple bilateral GGOs had been to some degree absorbed, but the fluid in the right thoracic cavity still increased ( 21 days after surgery) in Case 3 .

\section{CASE 3}

A 73-year-old man presented with a $10 \mathrm{~mm}$ diameter solid nodule on the dorsal segment of the right lower lobe on contrast CT images of the chest on January 3 (Figure 1, C1). He had 10-year history of hypertension and his pulmonary function testing was normal before surgery. He underwent thoracoscopic right lower lobectomy and was diagnosed as T1b N0 M0 lung adenocarcinoma on January 16 . His lymphocyte count decreased to $0.96 \times 10^{9} / \mathrm{L}$ on January 20 . Five days after operation, the patient presented cough and chest distress, and chest CT images displayed multiple, subpleural, small GGOs on bilateral lung (Figure 1,C2). He left hospital by himself for Chinese New Year. On January 22 , the patient had a fever with a temperature of $38.2^{\circ} \mathrm{C}$ and was readmitted and diagnosed with COVID-19 infection by RT-PCR for SARS-CoV-2 test. CT confirmed aggressive bilateral pneumonia on January 26 (Figure 1, C3). Blood lymphocyte count was $0.77 \times 10^{9} / \mathrm{L}$. He was administered moxifloxacin, oseltamivir, and interferon. On February 2, he had no fever and showed improvement of his bilateral pneumonia on CT images (Figure 1, C4). His lymphocyte count increased from $0.48 \times 10^{9} / \mathrm{L}$ (January 30) to $0.99 \times 10^{9} / \mathrm{L}$ (February 3 ). He gradually recovered and was discharged on March 2. 


\section{DISCUSSION}

We collected data on patients undergoing thoracoscopic lung surgery at ZhongNan Hospital of Wuhan University and Renmin Hospital of Wuhan University between January 1 and March 31, 2020, and found that 126 patients underwent lung lobectomies. During the same period, 3400 cases of SARS-CoV-2 were confirmed at the 2 hospitals. No patients with wedge resection, segmentectomy, and limited section contracted COVID-19 at this time. We had suspended lung surgeries since January 20 due to the outbreak of COVID-19 and Chinese New Year. Mortality of patients with no COVID- 19 was $<1 \%$ and perioperative mortality of patients with COVID-19 was $66.7 \%$. We only tested patients with typical symptomatic COVID-19 infection due to limited test kits. In addition, we did not fully understand this disease at this time. We obtained oral informed consent from patients and their family and the case study was approved by the institutional ethics board of Zhongnan Hospital of Wuhan University (No. 2020032). The patients in the 3 cases reported here had no symptoms of pneumonia before surgery. Case 1 and Case 3 were in the same room after surgery and may have been infected with COVID-19 by each other. They presented SARS-CoV-2 pneumoniarelated symptoms such as aggravated dyspnea, severe cough, and fever from 3 days to 6 days after their surgeries. These patients might have been in the incubation period of SARS-CoV-2 infection when they had their lung lobectomies. They most likely contract COVID-19 after surgery. It was too difficult to confirm the exact date of SARS-CoV-2 infection for these cases. Fortunately, no health care workers on this floor contracted COVID-19 except for 1 nurse.

There were common clinical characteristics for SARS$\mathrm{CoV}-2$ pneumonia among our case patients, such as fever, myalgia or fatigue, dry cough, radiographic bilateral patchy shadows or GGOs in the lungs, low or normal white-cell count or low lymphocyte count, and no alleviation of symptoms after 3-days of antimicrobial treatment. Severe lymphopenia was associated with worse outcome of patients with SARS-CoV-2 infection. ${ }^{1}$ On the CT manifestations of Case 1 and Case 3, the initial small patchy shadows of the lungs quickly developed into large bilateral lung shadows within 5 days, leading to respiratory failure rapidly. Postoperative pneumonia is a frequent complication after lung cancer surgery because common pneumonia frequently results from pathogenic bacteria. Patients have mortality ranges from $2 \%$ to $3 \%$. $^{2-}$ ${ }^{5}$ During this period, 30-day mortality was $1 \%$ in our 2 hospitals. However, 2 patients died of COVID-19 after lobectomy and 1 patient recovered from severe COVID-19 and survived. Patients in epidemic areas should receive chest $\mathrm{CT}$ and nucleic acid test for SARS-CoV-2 before lung surgery. Protective actions should be taken during the perioperative period and lung cancer surgeries should be delayed in patients potentially infected with SARS-CoV-2.

\section{CONCLUSIONS}

Mortality may be very high in patients infected with SARS-CoV-2 pneumonia after lung lobectomy. Lung surgery should be performed with extreme caution in SARSCoV-2 epidemic outbreak areas.

\section{References}

1. Wang D, Hu B, Hu C, Zhu F, Liu X, Zhang J, et al. Clinical characteristics of 138 hospitalized patients with 2019 novel coronavirus-infected pneumonia in Wuhan, China. JAMA. 2020;323:1061-9.

2. Paul S, Sedrakyan A, Chiu YL, Nasar A, Port JL, Lee PC, et al. Outcomes after lobectomy using thoracoscopy vs thoracotomy: a comparative effectiveness analysis utilizing the Nationwide Inpatient Sample database. Eur J Cardiothorac Surg. 2013;43:813-7.

3. Paul S, Altorki NK, Sheng S, Lee PC, Harpole DH, Onaitis MW, et al. Thoracoscopic lobectomy is associated with lower morbidity than open lobectomy: a propensity-matched analysis from the STS database. J Thorac Cardiovasc Surg. 2010;139:366-78

4. Kent M, Wang T, Whyte R, Curran T, Flores R, Gangadharan S. Open, video assisted thoracic surgery, and robotic lobectomy: review of a national database. Ann Thorac Surg. 2014;97:236-42.

5. Brioude G, Gust L, Thomas PA, D'Journo XB. Postoperative complications after major lung resection [Article in French]. Rev Mal Respir. 2019;36:720-37. 